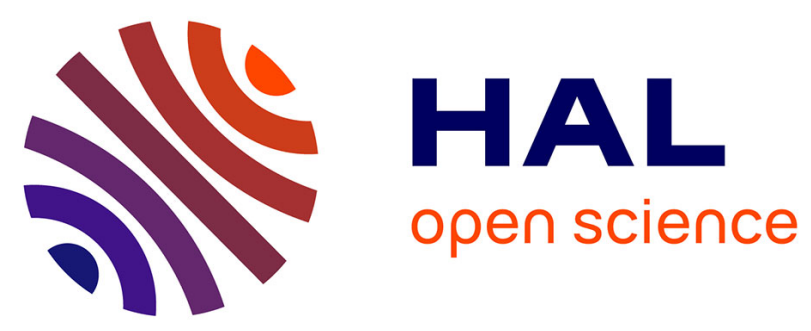

\title{
Theoretical and experimental optical studies of cholesteric liquid crystal films with thermally induced pitch gradients
}

Dimitrios C Zografopoulos, Emmanouil Kriezis, Michel Mitov, Corinne Binet

\section{- To cite this version:}

Dimitrios C Zografopoulos, Emmanouil Kriezis, Michel Mitov, Corinne Binet. Theoretical and experimental optical studies of cholesteric liquid crystal films with thermally induced pitch gradients. Physical Review E: Statistical, Nonlinear, and Soft Matter Physics, 2006, 73 (6), 10.1103/PhysRevE.73.061701 . hal-02887349

\section{HAL Id: hal-02887349 \\ https://hal.science/hal-02887349}

Submitted on 2 Jul 2020

HAL is a multi-disciplinary open access archive for the deposit and dissemination of scientific research documents, whether they are published or not. The documents may come from teaching and research institutions in France or abroad, or from public or private research centers.
L'archive ouverte pluridisciplinaire HAL, est destinée au dépôt et à la diffusion de documents scientifiques de niveau recherche, publiés ou non, émanant des établissements d'enseignement et de recherche français ou étrangers, des laboratoires publics ou privés. 


\title{
Theoretical and experimental optical studies of cholesteric liquid crystal films with thermally induced pitch gradients
}

\author{
Dimitrios C. Zografopoulos and Emmanouil E. Kriezis* \\ Department of Electrical and Computer Engineering, Aristotle University of Thessaloniki, Thessaloniki GR-54124, Greece \\ Michel Mitov ${ }^{\dagger}$ and Corinne Binet ${ }^{\ddagger}$ \\ Centre d'Élaboration de Matériaux et d'Études Structurales, CEMES, CNRS, Boîte Postale 94347, 31055 Toulouse Cedex 4, France
}

(Received 21 December 2005; published 6 June 2006)

\begin{abstract}
The reflection properties of cholesteric films with thermally induced pitch gradients are theoretically and experimentally studied. It is shown that the optical behavior of such films corresponds to the averaged contribution of a number of stochastic pitch variation profiles, due to the transversal and longitudinal nonuniformities that develop in the helical structure of such samples. Depending on the annealing time, both narrow-band and broadband behavior can be selectively achieved. The influence of the pitch profile gradient on the broadband reflection performance of cholesteric samples is theoretically analyzed, and a multi-slab structure for achieving optimum efficiency is proposed.
\end{abstract}

DOI: 10.1103/PhysRevE.73.061701

PACS number(s): 42.70.Df, 42.70.Qs, 61.30.Eb

\section{INTRODUCTION}

Cholesteric liquid crystals (CLCs) exhibit many remarkable properties due to the existence of a macroscopic helical structure $[1,2]$. Iridescent selective reflection colors are displayed by a uniformly oriented Grandjean planar texture, i.e., when the helical axis is perpendicular to the observation plane. Inside a small wavelength band $\Delta \lambda$ centered around $\lambda_{0}$, an incident light beam parallel to the helical axis is split into two circularly polarized components: One of which is simply transmitted whereas the other is totally reflected. The rotation of the reflected circularly polarized light agrees with the screw sense of the structure. At normal incidence, maximum reflection occurs when the incident wavelength $\lambda_{0}$ matches the following Bragg law:

$$
\lambda=\lambda_{0}=n P,
$$

where $n=\left(n_{o}+n_{e}\right) / 2$ is the average of the ordinary $\left(n_{0}\right)$ and extraordinary $\left(n_{e}\right)$ refractive indices of the locally uniaxial structure and $P$ is the helical pitch. It is in the region $\Delta \lambda$ of the pitch band that most of optical investigations of CLCs have concentrated. The reflection band $\Delta \lambda$ is given by the following relation:

$$
\Delta \lambda=P \Delta n,
$$

where $\Delta n=n_{e}-n_{o}$ is the birefringence. An incident beam with a wavelength out of $\Delta \lambda$ is simply transmitted.

However huge the choice for pitch amplitude is, $\Delta n$ is typically limited to values below 0.3 for colorless organic materials. As a consequence, $\Delta \lambda$ is commonly less than $100 \mathrm{~nm}$ in the visible spectrum and is more often equal to $50 \mathrm{~nm}$. Selective behavior is desired for several families of

\footnotetext{
*Electronic address: mkriezis@auth.gr

${ }^{\dagger}$ Electronic address: mitov@cemes.fr

${ }^{\ddagger}$ Now at: Laboratoire de Dynamique et Structure des Matériaux Moléculaires, UMR 8024 CNRS, UST Lille, F-59655 Villeneuve d'Ascq, France.
}

applications-optical filters, thermal imaging, laser or paint technologies-but a drawback lies in the fact that the known CLC filters have a reflection bandwidth that is too limited for specific purposes, like full color or white or black reflective displays, broadband circular polarizers or smart windows in buildings, where a control over the solar spectrum is desirable. Consequently, and from fundamental principles, the consideration of elaboration schemes leading to CLC materials with unusual helical structures is required to have access to enlarged reflection bandwidths.

\section{A. State of the art}

\section{Nonswitchable CLCs}

Most studies deal with a CLC polymer network with a pitch gradient in the visible spectrum that requires an illumination gradient during a UV-crosslinking reaction [3-7]. As a consequence, $\Delta \lambda$ can result in values greater than $300 \mathrm{~nm}$. In the pioneering work done by Philips group $[3,4]$, the pitch gradient is due to a photoinduced diffusion during a UVpolymerization reaction occurring in a chiral and nematic monomers blend with different UV-reactivities. An absorbing dye is required to create a UV-intensity gradient over the film thickness. Such broadband reflectors greatly improve the light yield (by $40 \%$ in [5]) and the energy efficiency of LC devices by recycling wrongly polarized light in the backlight system $[5,6]$. The broadening of the reflection band and the enhancement of polarized backlight output have been studied for two types of CLC films [8-13]: (i) A stack of red-, green-, and blue-reflecting films [8-10]; and (ii) a single layer [11-13] that achieves its wide selective reflection band because of its engineered pitch distribution [12]. We experienced an alternative route for increasing $\Delta \lambda$ which led to a broadband CLC with a pitch gradient $[14,15]$. By contrast to previous studies, the process depends on a thermal diffusion between two CLC oligomer films with different chiralities and the pitch gradient is the consequence of a concentration gradient which is afterwards frozen by quenching. Neither 


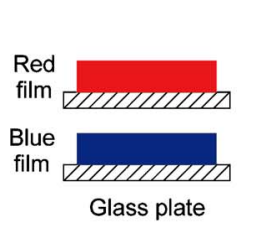

Step 1

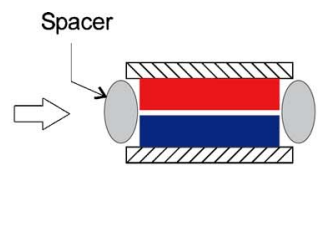

Step 2

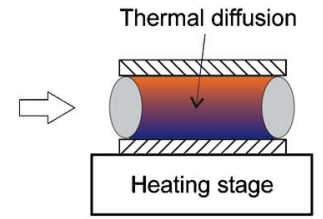

Step 3

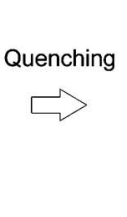

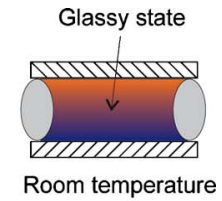

Step 4
FIG. 1. (Color online) Elaboration process for the annealing of the cholesteric films. polymerization reaction nor photoinduced phase separation is involved. Besides, the reflector is a single-layer CLC film in the glassy state. The reflection band may simply be tuned by varying the annealing time and different optical properties (like tunable bandwidths) are available [16]. The pitch gradient was demonstrated and evaluated by transmission electron microscopy (TEM) investigations of cross sections [17]. The glassy state of the reflector was discussed as offering new opportunities for optical data storage. Spatially modulated photoracemization in glassy chiral nematic films can also generate a pitch gradient in a CLC structure [18]. Furthermore, we adapted the thermally induced pitch gradient from the case of glassy CLCs to the CLC gel one, which can be addressed by an electric field [19]. The basic idea lied in the association of two individual polymer-stabilized CLC (PSCLC) layers the interdiffusion of which is controlled by UV-curing. In the case of CLC-polymer composites with high concentrations in polymer, an approach that lies in a thermally induced pitch variation simultaneously carried out with the UV-crosslinking reaction was also developed in our laboratory [20].

\section{Switchable CLCs}

Studies in PSLCs correlate the influence of the chemical structure of one or several compounds of the mixture in the $\Delta \lambda$ increase [21-23]. The fact of combining chiral tolane mesogenic side-on additives into the blend shows an improvement in birefringence and consequently increases $\Delta \lambda$ [21]. Due to a greater chiral concentration in the vicinity of the polymer network than in the bulk of a PSCLC with specific chiral additives, a distribution of two areas with different pitches is evoked to explain a reflection broadening (overlap in the reflection spectra from these two areas) [22]. The position and stereochemical configuration of the chiral centers in the side group of functionalized polymers, with respect to the backbone, play an integral role in the observed reflection spectra [23]. The value $\Delta \lambda$ can also be increased in PSCLCs when compounds referred to as excited-state quenchers are added to the monomeric mixtures [24,25]. Such quenchers work by transferring the energy of the excited state of the initiator to themselves and by absorbing UV-light. A phase separation leading to concentration fluctuations corresponding to different pitches is expected. The latest methods leading to switchable broadband gap CLC reflectors we developed involve memory effects due to a thermally induced pitch variation which occurred during the polymer network formation [26,27].

\section{B. Objectives of the present studies}

We focus our study to the case of cholesteric glassy materials whose spectral characteristics evolve from a selective to a broadband film exhibiting light reflection over several hundreds of nanometers, and investigate their optical properties both experimentally and theoretically. Different optical properties, from a narrow-band gap to a broadband one can be exhibited by tuning the annealing time. The inverse transition, from a broad- to a narrow-band gap, is also demonstrated. Such a process is driven by one parameter: The annealing time. The experimental results are compared with numerical simulations, assuming that the optical response of the cholesteric films corresponds to many stochastic pitch variation profiles. Finally, we give theoretical insights to optimize the broadband reflective state of cholesteric films with pitch gradients.

\section{EXPERIMENTAL PART}

\section{A. Materials}

Cholesteric liquid crystalline oligomers were chosen. The CLC molecule is a siloxane cyclic chain with two types of side-chains attached via spacers: A nonchiral mesogen and a chiral one [28]. When coated on a glass or plastic substrate, the compound shows typical iridescent colors ranging from blue to red simply tuned by the molar percentage of chiral mesogens in the oligomer molecule, which varies from $50 \%$ to $31 \%$. The cholesteric phase appears between $180 / 210{ }^{\circ} \mathrm{C}$ (isotropic transition) and $40 / 50{ }^{\circ} \mathrm{C}$ (glass transition temperature). A significant advantage regarding the scope of our studies is that these materials can be very easily quenched at room temperature and the cholesteric structural order with its color properties are then permanently stored within a solid film.

\section{B. Material design}

The elaboration path is summarized in Fig. 1 and is basically associated to the idea of an anisotropic diffusion between two compounds with different chiralities. Step 1.$40 \mu \mathrm{m}$ thick films of blue and red compounds are spread with a handcoater onto two distinct $1 \mathrm{~mm}$ thick glass plates without any alignment layer. Step 2-A sandwich-cell is made with these blue and red films separated with $19 \mu \mathrm{m}$ calibrated spacers. Step 3-The cell is sealed and kept at $85^{\circ} \mathrm{C}$. This temperature corresponds to a stable cholesteric phase for which the materials are viscous. Consequently, a diffusion process between the red and blue compounds-and therefore a concentration gradient-may occur in a direction perpendicular to the plane of films. Since these materials belong to the same molecule family, high miscibility is reached. Step 4-After such an annealing process, the cell is swiftly put on a metallic substrate at room temperature. The macroscopic characteristics of the film evolve from a viscous 


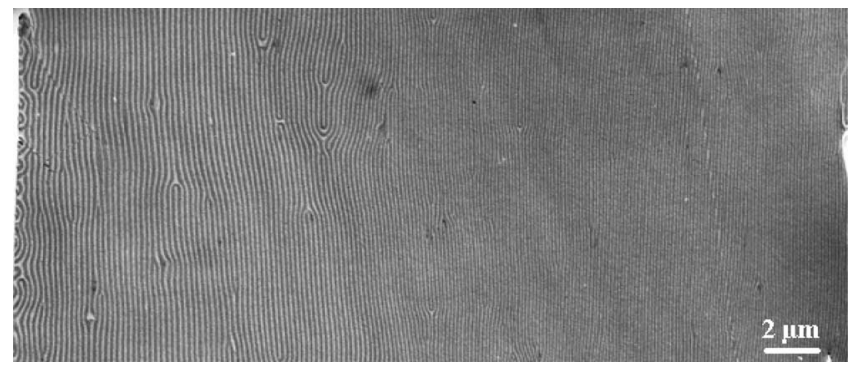

FIG. 2. TEM micrograph of a cholesteric sample after an annealing procedure of $25 \mathrm{~min}$ duration. As noticed, the process leads to a continuous pitch gradient between the two original individual films due to thermal diffusion.

to a glassy state. Such a cooling prevents crystallization and preserves the cholesteric structure. The quenching procedure is not affected by the fact that the cholesteric compounds have slightly different glassy transitions (due to the different number of cholesterol-containing side-chains), since room temperature $\left(20-25{ }^{\circ} \mathrm{C}\right)$ is well below their transition temperatures and the quenching time is sufficiently quick. Figure 2 shows a TEM micrograph of a cross section of the film after an annealing time equal to $25 \mathrm{~min}$. The former bilayer film is now a homogeneous single-layer film, due to the efficiency of the diffusion process. The cholesteric film presents a contrast of dark and bright lines. The distance between two consecutive dark (or bright) lines is about half of the pitch value; the helical axis is everywhere perpendicular to the lines. This periodic pattern clearly indicates that a continuous pitch gradient forms along the direction perpendicular to the film surfaces, which confirms the concept of a cholesteric structure with a gradient pitch. In addition to the above process, lateral material flow also takes place; however, the time scale of such flow is short in comparison with the time which is required for the progressive building of the pitch gradient. As a consequence, the lateral homogeneity is not significantly degraded, as it can be verified from Fig. 2 .

\section{Methods}

The investigation of light transmittance properties of the materials at room temperature is carried out by using unpolarized UV-visible light spectroscopy (UV-3100 Shimadzu). When a thermally controlled investigation of transmittance is required, the cell is put in a Mettler-Toledo heating stage and the spectrophotometer used is the HR2000CG-UV-NI from Ocean Optics, associated to an optical microscope. All baselines were done with air in the reference beam path. The sample in Fig. 2 was observed on a TEM Philips CM12 at room temperature and under normal conditions (see [17] for more details on the material preparation for TEM investigations).

\section{THEORETICAL STUDIES FOR LIGHT PROPAGATION}

\section{A. Numerical model}

The optical properties of the cholesteric films were theoretically studied by means of the finite-difference time- domain (FDTD) method, a well-known simulation tool capable of dealing with light propagation through anisotropic materials [29-31]. Given that we examine the case of normal incidence, a one-dimensional grid is sufficient to describe the spatial periodic variation of a cholesteric film's dielectric tensor. The $D_{u}, E_{u}, H_{u}(u=x, y)$ field components are discretized over the one-dimensional Yee grid, while $D_{z}=E_{z}$ $=H_{z}=0$, since we assume propagation along the $z$-axis. At the beginning of each time step the components of the dielectric displacement are calculated from previously stored values via

$$
\left.[\boldsymbol{D}]\right|_{k} ^{n+1 / 2}=\left.[\boldsymbol{D}]\right|_{k} ^{n-1 / 2}+\left[\begin{array}{cc}
0 & -c_{D} \\
c_{D} & 0
\end{array}\right]\left(\left.[\boldsymbol{H}]\right|_{k} ^{n}-\left.[\boldsymbol{H}]\right|_{k-1} ^{n}\right),
$$

where $c_{D}=\Delta t / \Delta z, \Delta z$ and $\Delta t$ being the spatial and temporal discretization steps, respectively, and the notation $\left.F_{u}\right|_{k} ^{n}$ $=F_{u}(k \Delta z, n \Delta t)$ has been adopted. The values obtained by Eq. (3) yield the values of the electric field components $E_{x}, E_{y}$ via $\boldsymbol{E}=\widetilde{\varepsilon} \boldsymbol{D}$, where $\widetilde{\varepsilon}$ is the dielectric tensor. For a cholesteric liquid crystal material of pitch $P$ and ordinary and extraordinary refractive indices of $n_{o}$ and $n_{e}$, the dielectric tensor is calculated by [32]

$$
\widetilde{\varepsilon}(z)=\varepsilon_{0}\left[\begin{array}{cc}
b+a \cos (2 q z) & a \sin (2 q z) \\
a \sin (2 q z) & b-a \cos (2 q z)
\end{array}\right],
$$

where $a=\left(n_{e}^{2}-n_{o}^{2}\right) / 2$ is a measure of the optical dielectric anisotropy, $b=\left(n_{e}^{2}+n_{o}^{2}\right) / 2$ a measure of the average optical dielectric constant and $q=2 \pi / P$ is the spatial frequency of the helical rotation of the director. The magnetic field is then updated at the end of the time step using

$$
\left.\left.[\boldsymbol{H}]\right|_{k} ^{n+1}=\left.[\boldsymbol{H}]\right|_{k} ^{n}+\left[\begin{array}{cc}
0 & c_{H} \\
-c_{H} & 0
\end{array}\right]([\boldsymbol{E}]]_{k+1}^{n+1 / 2}-\left.[\boldsymbol{E}]\right|_{k} ^{n+1 / 2}\right),
$$

where $c_{H}=\Delta t /\left(\mu_{0} \Delta z\right)$ and $\mu_{0}$ is the free-space magnetic permittivity.

The grid is excited by an incident Gaussian modulated pulse whose spectral content covers the frequency range of interest. The pulse is allowed to propagate through the cholesteric film, while a fast Fourier transform (FFT) of the time samples at the output yields the transmitted spectrum. The polarization (linear, circular, or elliptical) of the incident field is controlled by properly selecting the magnitude and phase of the incident $E_{x}$ and $E_{y}$ components. Finally, the computational space is backed by the PML absorbing boundary condition in order to eliminate any reflections that could undermine the accuracy of the calculations.

\section{B. Simulations}

The implementation of the FDTD model described above aims at a theoretical comparison for the experimentally measured transmission curves of the cholesteric films described in Sec. II. This one-dimensional model, though, is valid only for ideal films, i.e., under the assumption that the helical pitch variation profile is uniform along the $x, y$ directions. This idealization has been proven far from being valid in practice; the cholesteric films after the heating procedure showed both transversal variations and, additionally, a dis- 
turbed longitudinal profile as evidenced by Fig. 2. Clearly, the exhibited inhomogeneities prevent the uniformity of the films and locally modify the values of the helical pitch in a random way, thus, rendering impossible the simulation of the film's pitch optical response by a single one-dimensional pitch profile.

Nevertheless, given that these structural irregularities cannot be attributed to determinable factors, such as surface treatment, interfaces between different materials, etc., it should be consistent to consider the overall optical behavior of the cholesteric film as the average of the contribution of stochastic one-dimensional samples, where the pitch values are allowed to deviate around a mean pitch transition curve. Indeed, TEM microscopy verifies this assumption ([17], Fig. 2 ); the pitch values for the red film were found to deviate $40 \mathrm{~nm}$ around the mean value $P_{R}=475 \mathrm{~nm}$, while in the case of the blue film a deviation of $20 \mathrm{~nm}$ around $P_{B}=295 \mathrm{~nm}$ was observed. Moreover, the slit size $(1 \mathrm{~mm})$ of the spectrometer used for the extraction of the experimental curves is orders of magnitude greater than the scale of the inhomogeneities taking place in the cholesteric film. Consequently, the curves obtained experimentally represent the cumulative average of numerous stochastic samples, rather than a single indicative pitch transition profile.

Taking into account this fact, three layouts were investigated for the $5 \mathrm{~min}, 25 \mathrm{~min}$, and $83 \mathrm{~h} 25 \mathrm{~min}$ annealing duration cases, respectively. The ordinary and extraordinary refractive indices of the cholesteric materials considered here were assessed to be $n_{o}=1.4$ and $n_{e}=1.6$. These values were estimated based on the experimental observations regarding the distribution of the pitch value and the optical transmission spectra. Nevertheless, during the preparation of the samples for TEM investigations (ultramicrotomy), it is not always possible to expect cutting the sample in a direction exactly perpendicular to the helical axis and, in addition, the cut-direction with respect to the axis orientation may vary from one sample to another. For these reasons, it has to be anticipated that the periodicity values as extracted from TEM micrography are to an extent overestimated, with an error margin of roughly $5 \%$. This error leads to a small underestimation of the refractive indices.

A set of 100 stochastic samples was used for each case; typical members for all three cases are shown in Fig. 3. Since the experiments were conducted with unpolarized incident light, an average optical power transmission coefficient $\bar{T}$ defined as

$$
\bar{T}=\frac{1}{2}\left(\left|T_{x x}\right|^{2}+\left|T_{x y}\right|^{2}+\left|T_{y x}\right|^{2}+\left|T_{y y}\right|^{2}\right),
$$

was extracted for every sample examined, $T_{i j}$ being the amplitude transmission coefficient of $i$-polarized light assuming incident light of $j$-polarization [33]. The pitch transition for the 5 and 25 min cases was considered linear between the blue and red films, while for the $83 \mathrm{~h} 25 \mathrm{~min}$ case the cholesteric film was considered homogeneous with a mean pitch value of $P_{H}=345 \mathrm{~nm}$ and a deviation of $25 \mathrm{~nm}$. The distribution of the random pitch values for all cases examined was considered to be Gaussian.

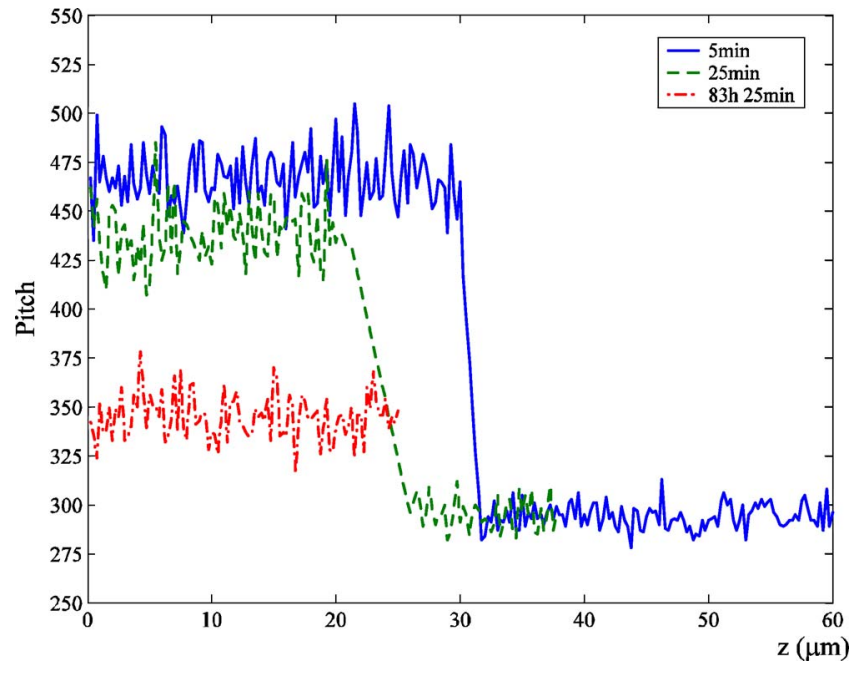

FIG. 3. (Color online) Typical stochastic pitch variation curves for the three cases under study: $5 \mathrm{~min}, 25 \mathrm{~min}$, and $83 \mathrm{~h} 25 \mathrm{~min}$ annealing duration. The thickness of the cholesteric film reduces for longer annealing times, from $60 \mu \mathrm{m}(5 \mathrm{~min})$ to $38 \mu \mathrm{m}$ (25 min) and $25 \mu \mathrm{m}$ (83 h $25 \mathrm{~min})$.

Before moving on to the simulation results, it would be supplementary to make some remarks on some specific issues regarding the pitch profiles of Fig. 3; as it can be readily observed, the thickness of the cholesteric film depends on the duration of the annealing procedure. In the $5 \mathrm{~min}, 25 \mathrm{~min}$, and $83 \mathrm{~h} 25 \mathrm{~min}$ annealing time cases the corresponding thicknesses observed were approximately 60, 38, and $25 \mu \mathrm{m}$. Although the pitch gradient is formed along the $z$ direction, the liquid crystal material also diffuses flowing on the transverse plane; consequently, the total thickness gradually diminishes as the thermal diffusion process lasts longer. Another point of interest concerns the pitch of the red film for the three cases. The red film's pitch was found to be 465 and $440 \mathrm{~nm}$ after 5 and $25 \mathrm{~min}$ of annealing, respectively. Thus, it seems that the heating of the red film has an impact on its pitch value - for longer heating times the pitch value reduces. On the other hand, the blue film retains its pitch value regardless of the annealing time. This particular property of the red films also accounts for the fact that in the $83 \mathrm{~h}$ 25 min case the homogeneous pitch value observed was $P_{H}=345 \mathrm{~nm}$, which is closer to the blue film pitch, staying below the average value $\left(P_{R}+P_{B}\right) / 2=385 \mathrm{~nm}$. Such a physical behavior could probably be related to the fact that the viscosity of the red film is lower than the viscosity of the blue one at the same temperature. This difference in macroscopic viscosity values between the red and blue films is related to the number of cholesterol groups in one of the two kinds of side-chain in the oligomer molecule. The red compound is more fluid. Apart from the variation of the red film's pitch, this physical property also elucidates the origin of the following two observations: (i) The interface between the two pristine films is not be kept at the half-width of the sandwich film after the annealing has started; (ii) the confinement ratio (thickness over pitch ratio) is not the same from the top of the cell to the bottom and we can expect that the helix is more frustrated in the "red part" than in the "blue one." 


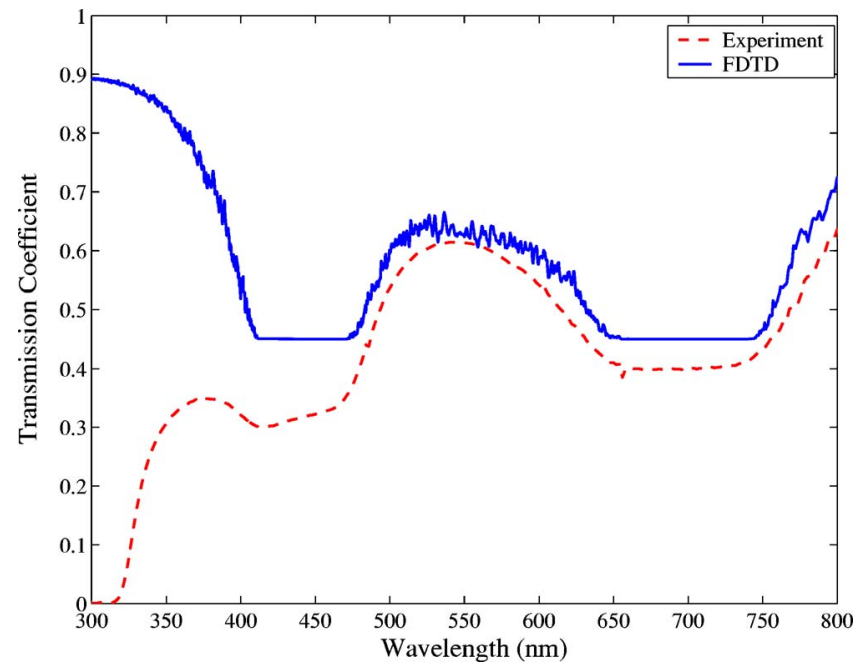

FIG. 4. (Color online) Transmission spectra for the 5 min case: Experimental curve and theoretical estimation. The pitch gradient has not yet been formed. The transmission spectrum corresponds to two individual narrow-band cholesteric films.

The simulation results of the stochastic samples were averaged for each one of the three cases of interest, yielding, thus, the theoretical transmission curves. Their values were corrected in order to account for the Fresnel reflections on the two glass plates supporting the cholesteric films. The final transmission curves, both theoretical and experimental, are shown in Figs. 4-6. It is emphasized that this particular study has dealt only with the normal incidence case, both from the experimental as well as from the theoretical point of view. In the case of oblique incidence it is anticipated that the reflection band will experience a blue-shift and Eq. (1) will now read $\lambda_{0}=n P \cos \theta, \theta$ being the propagation angle measured inside the liquid crystal, while the reflection band $\Delta \lambda$ should remain unaffected [34].

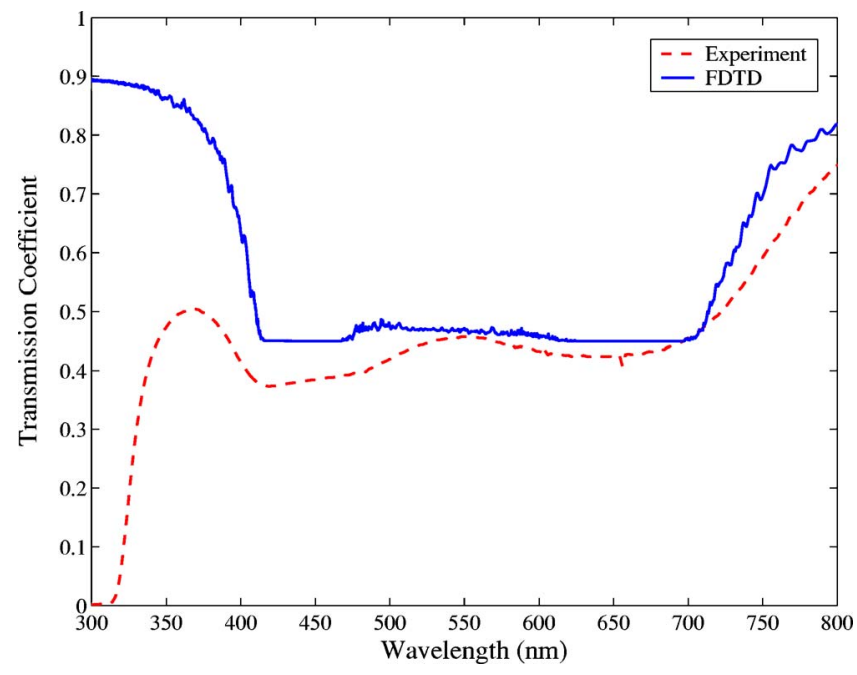

FIG. 5. (Color online) Transmission spectra for the 25 min case: Experimental curve and theoretical estimation. The pitch gradient formed along the sample leads to broadband reflective behavior.

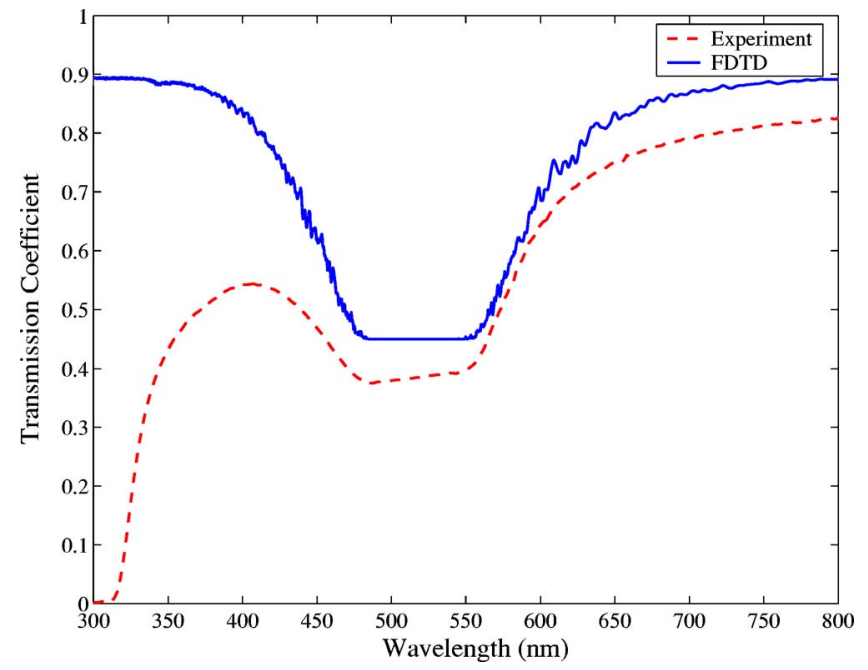

FIG. 6. (Color online) Transmission spectra for the 83 h 25 min case: Experimental curve and theoretical estimation. Homogeneity of the pitch value over the entire film has been reached leading to narrow-band reflection (green-film).

\section{Discussion}

The transmission curves depicted in Figs. 4-6 permit a direct comparison between theoretical and experimental results. The reflective behavior of the processed films for the 5 and 25 min cases is exhibited for both theoretical and experimental curves. Accordance is also evident for the $83 \mathrm{~h}$ 25 min case, where the homogeneity of the cholesteric film leads to narrow-band reflectivity. The bandgaps resulting from the simulations are located in the same regions and they spread almost over the same spectral width as in the experimental curves. The averaging used for the extraction of the simulation curve yielded smooth curves without significant rippling that would be inevitably present in the case of simulating the pitch transition of a single profile. Thus, the selection of the pitch variation profiles of Fig. 3 proved consistent with the experimental reality of the phenomenon.

Another point of significant interest is the gradual decline of the transmission spectra towards shorter wavelengths, which is observed in the experimental curves. Although the abrupt roll-out to zero in all transmission spectra at the wavelength threshold of $320 \mathrm{~nm}$ is due to the glass plate cut-off wavelength, for the rest of the spectral zone this decline is clearly attributed to some dispersive loss mechanisms, which were not considered in the FDTD simulations. Nevertheless, in an attempt to elucidate the origins of these losses we have conducted a supplementary experiment; a cholesteric film with an enlarged reflection bandwidth (annealing time $=25 \mathrm{~min}$ ) was gradually heated and the film's transmission spectra were extracted at various temperature levels. Figure 7 shows the results of this experiment.

As the temperature increases, the reflection bandgap of the cholesteric film shrinks, since the values of the refractive indices $n_{o}$ and $n_{e}$ both tend towards the value $n_{i s o}$ (the refractive index of the LC material in the isotropic case), and, mainly, the concentration gradient is progressively disappearing (homogeneity is progressively reached), while the 


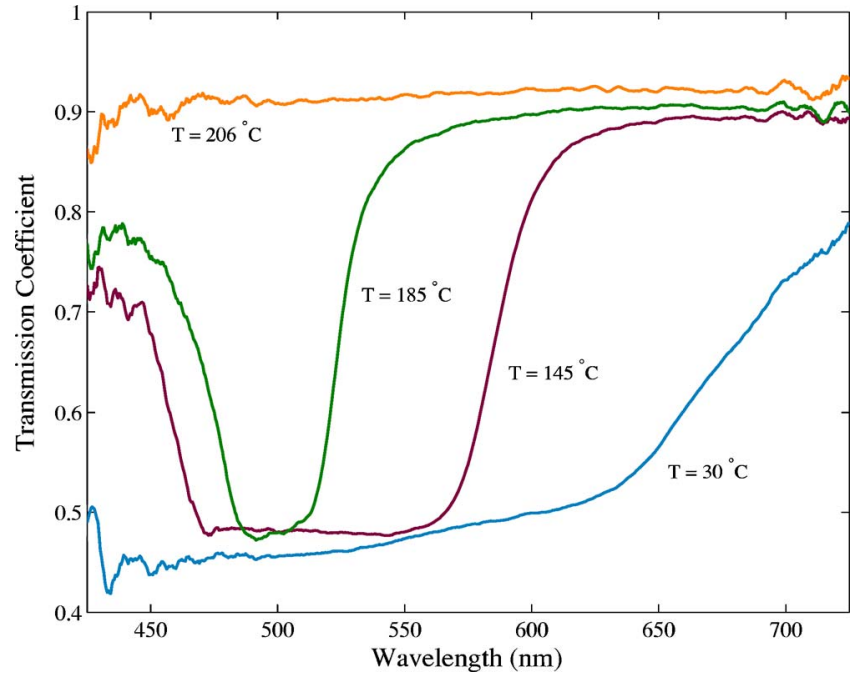

FIG. 7. (Color online) Impact of the heating temperature on the reflective behavior of a cholesteric film with a broadened reflection bandwidth (annealing time $=25 \mathrm{~min}$ ).

optical properties undergo a thermally induced broadband to narrow-band transition.

The fainting of the reflective behavior is also accompanied by a reduction of the overall losses; the transmission spectrum for the $206{ }^{\circ} \mathrm{C}$ case, where the film is clearly in the isotropic state, shows no wavelength-dependent losses whatsoever. The $90 \%$ ceiling value is due to the Fresnel reflection of the glass plates supporting the film. Conclusively, this confirms that the loss mechanism is not due to some intrinsic absorption mechanism of the LC material, but could be most probably attributed to scattering losses between the numerous domain walls and rugged surfaces transversely formed along the untreated cholesteric sample; clearly, this multidomain structure could not be numerically simulated due to the lack of a deterministic model on the formation of such structural walls and domains.

\section{THEORETICAL INSIGHTS ON THE OPTIMIZATION OF THE BROADBAND REFLECTIVE BEHAVIOR OF CHOLESTERIC FILMS WITH PITCH GRADIENT}

We have considered so far how a thermally induced linear pitch variation between two cholesteric films can lead to a broadband reflective behavior. In the present section we attempt a general approach towards the determination of an optimized pitch transition curve for the case of cholesteric broadband reflectors.

Let us assume two cholesteric films with pitch values $P_{B}$ and $P_{R}$ for the blue and red film respectively, whose thicknesses $d_{B}, d_{R}$ correspond to the same number of pitch periods, and an intermediate zone of thickness $L$, where any pitch transition curve from $P_{B}$ to $P_{R}$ is allowed. The form of the transition curve determines the distribution of the disposed space over the intermediate pitch value range $P_{B}<P$ $<P_{R}$, which is responsible for the broadband reflection. In the examples studied we considered the following indicative family of curves
TABLE I. Values of the structural parameters used for the studied cases $\mathrm{A}$ and $\mathrm{B}$.

\begin{tabular}{lcc}
\hline \hline Parameter & Case A & Case B \\
\hline$P_{B}$ & $250 \mathrm{~nm}$ & $250 \mathrm{~nm}$ \\
$P_{R}$ & $500 \mathrm{~nm}$ & $500 \mathrm{~nm}$ \\
$d_{B}$ & $5 \mu \mathrm{m}$ & $5 \mu \mathrm{m}$ \\
$d_{R}$ & $10 \mu \mathrm{m}$ & $10 \mu \mathrm{m}$ \\
$L$ & $9 \mu \mathrm{m}$ & $4.5 \mu \mathrm{m}$ \\
$n_{o}$ & 1.45 & 1.4 \\
$n_{e}$ & 1.55 & 1.6 \\
\hline \hline
\end{tabular}

$$
P(z)=P_{B}+\left(P_{R}-P_{B}\right)\left(\frac{z}{L}\right)^{n}, \quad n>0 .
$$

Although there is an infinity of possible curves, this particular set is rather representative, since by controlling the parameter $n$, one can obtain any distribution of the total thickness $L$ over the interested range of pitch values; for $n=1$ follows a linear transition, while for $n<1(n>1)$ the pitch values closer to the red (blue) region are promoted.

Two specific cases (A and B) were studied; the parameters used in both cases are given in Table I. The thicknesses of the blue and red films equals to 20 periods which is sufficient enough to ensure that the two films will form two welldefined bandgaps centered at $\lambda_{B}=\bar{n} P_{B}=375 \mathrm{~nm}$ and $\lambda_{R}$ $=\bar{n} P_{R}=750 \mathrm{~nm}$ for both cases.

The optical properties of the cholesteric layouts for diverse values of the order $n$ were investigated via FDTD simulations. In Fig. 8 three indicative transmission spectra are presented for Case A. The severe bandgaps centered at $\lambda_{B}, \lambda_{R}$ emerge due to the reflective behavior of the two basic films. In the intermediate zone, though, great diversification is observed; for $n=1 / 3(n=3)$ the spectral area around $\lambda_{R}$ $\left(\lambda_{B}\right)$ clearly shows greater reflectivity than at the opposite edge of the zone, due to the spatial distribution which favors

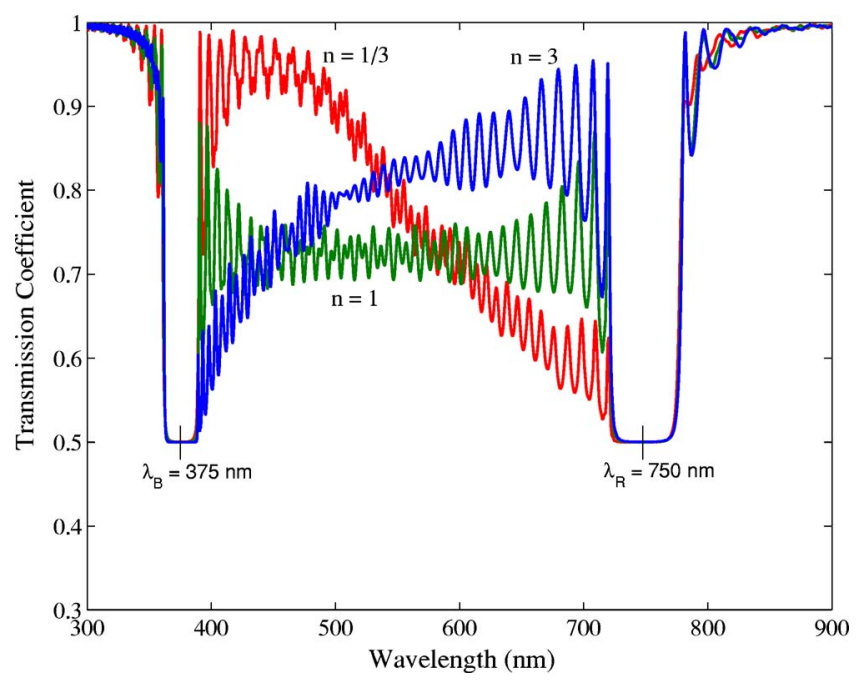

FIG. 8. (Color online) Transmission spectra $\bar{T}(\lambda)$ for three curves $(n=1 / 3, n=1, n=3)$ for the first case (case A). 


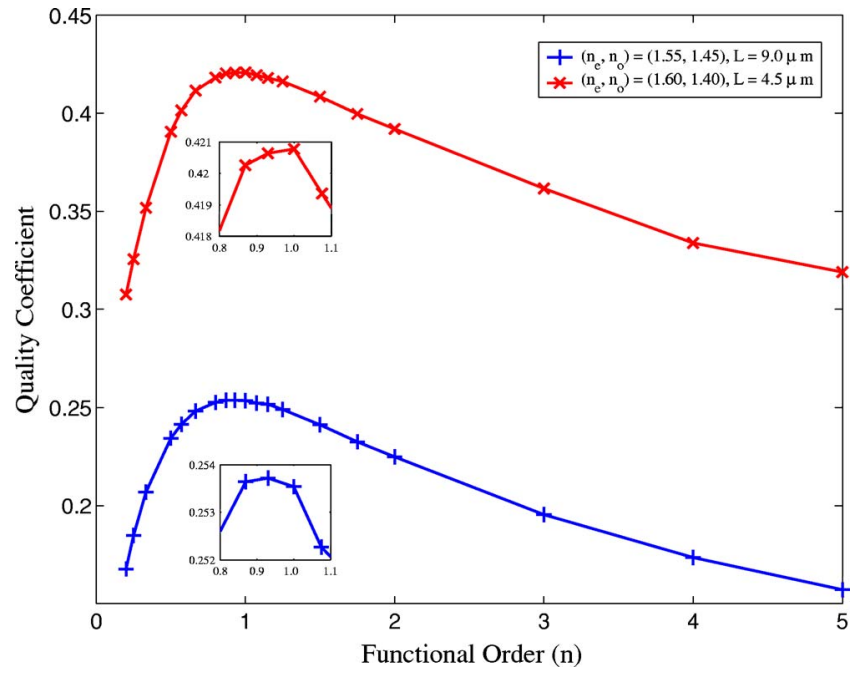

FIG. 9. (Color online) Quality coefficient $\widetilde{R}$ over $n$ for case A "+"s and case B " $\times$ "s. The insets show a magnification of the plots for values of functional order $n$ around $n=1$.

higher (lower) pitch values. On the contrary, for $n=1$ the transmission spectrum seems well-balanced.

The reflective properties resulting from the variation of the transition curve were assessed by means of a quality coefficient

$$
\widetilde{R} \equiv 1-\frac{1}{\Delta \lambda} \int_{\lambda_{B}}^{\lambda_{R}} \bar{T}(\lambda) d \lambda, \quad \Delta \lambda=\lambda_{R}-\lambda_{B}
$$

which represents the average power reflection coefficient over the broadband wavelength zone defined by $\lambda_{B} \leqslant \lambda$ $\leqslant \lambda_{R}$. Ideally, one would expect $\widetilde{R}=0.5$ for a perfect broadband reflector and values closer to zero for more moderate performances. In the more general case of oblique incidence, it is anticipated that this quality coefficient will drop substantially, due to the expected blue-shift of the reflection band. The quality factor resulting from the simulations for both cases is presented in Fig. 9.

Figure 9 shows that both cases show similar dependence of the quality factor over $n$; the optimum quality coefficient was found for $n=0.93, \widetilde{R}_{\max }^{A}=0.254$ in the first case, and $n$ $=1, \widetilde{R}_{\max }^{B}=0.421$ for the second. For $0.8<n<1.1$ the quality coefficient drops less than $1 \%$ from its peak value, a reduction that could be considered negligible, since, in practice, other factors (absorption, scattering, etc.) have a definitely greater impact on the reflective behavior of the cholesteric films. Thus, the linear transition curve proves a favorable selection among the family of transition curves studied. In Fig. 9 it is also clearly evidenced that the quality coefficient is substantially higher for case B rather than case A. Since $\Delta n^{B}=2 \Delta n^{A}$ and $L^{B}=0.5 L^{A}$ it is concluded that the value of $\Delta n$ influences the optical performance of the films in a greater extent than the thickness of the transition zone $L$.

Since the family of curves of Eq. (7) can in general account for possible yet smooth pitch transitions we additionally simulated a special transition case which was extracted relying on some insight of the physical properties of the

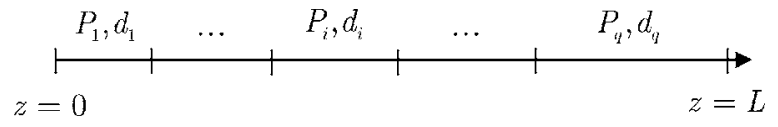

FIG. 10. The transition zone is distributed among a finite number of slabs with constant pitch values and constant number of pitches per slab.

cholesteric films. The thickness $L$ of the transition zone is allowed to spread over $q$ slabs of constant pitches $P_{i}, i$ $=1, \ldots, q$, and thicknesses $d_{i}, i=1, \ldots, q$ (Fig. 10). Each slab acts as an isolated cholesteric film yielding a bandgap that covers the spectral zone $n_{o} P_{i}<\lambda<n_{e} P_{i}$, assuming $n_{e}>n_{o}$. Most favorably, the discrete bandgaps of the $q$ slabs should cover the entire of the spectral range $n_{e} P_{B}<\lambda<n_{o} P_{R}$ and take full advantage of the space provided. The condition $P_{i}$ $=k^{i} P_{B}, k=n_{e} / n_{o}$ ensures that the whole spectral range of interest is covered without any overlapping bandgaps that might lead to unjustifiable losses of space. Furthermore, the acuteness of the reflection band for a cholesteric slab depends on the number of pitch periods that fit into the slab's thickness, and not the value of the thickness itself; thus, in the case of a series of adjacent reflection bands that we examine, an equal number $c$ of pitches $P_{i}$ should be addressed to each slab, so that the total thickness of the transition zone is optimally exploited $\left(d_{i}=c P_{i}\right)$. Conclusively, the following conditions must be met

$$
\sum_{i=1}^{q} d_{i}=L, \quad P_{i}=k^{i} P_{B}, \quad d_{i}=c P_{i}, \quad P_{q}=P_{R} / k .
$$

These conditions lead to the following values for the parameters $q$ and $c$ :

$$
q=\frac{\ln \left(P_{R} / P_{B}\right)}{\ln k}-1, \quad c=\frac{L}{P_{B}} \cdot \frac{1-1 / k}{\left(k^{q}-1\right)} .
$$

The parameters $q$ and $c$ need not be constrained to integers; the number of pitch periods per slab may be real as long as no abrupt phase jumps are observed between adjacent films that might lead to the manifestation of defect modes [35]. Additionally, the distribution of slabs in the general case where $q \in \mathrm{R}^{+}$may be interpreted as a series of $[q]$ slabs with thicknesses $d_{i}, i=1, \ldots,[q]$ and a final slab whose thickness is given by

$$
d_{[q]+1}=L-\sum_{1}^{[q]} d_{i}=c(q-[q]) P_{[q]+1},
$$

[q] denoting the integer part of $q$.

In order to obtain comparative results, we simulated the slab-transition case for the parameters of case B examined above, with $q=4.19$ and $c=3$ being the values obtained by Eq. (10). The simulation yielded a value of 0.4355 for the quality factor, which is $\sim 3.5 \%$ greater than the optimum value of case B. Although these discrete slabs cannot be formed by processes such as thermal diffusion, or polymerization phenomena, they might be fabricated by means of chiral film deposition techniques over rotating substrates, such as those referred in [36-38]. 


\section{CONCLUSIONS}

Broadening the light reflection bandwidth can be realized by a simple thermal treatment of a cholesteric glassy film, with a reduced number of independent driving parameters. The position of the wavelength band in the light spectrum depends on the choice of two compounds differing only in their chirality. The bandwidth is simply tuned by the annealing time. The inverse transition, from a broad- to a narrowband optical filtering, has also been demonstrated. The optical properties of these films were studied both experimentally and theoretically. The experimental curves were found in excellent agreement with numerical simulations, assuming that the optical response of the cholesteric films corresponds to many stochastic pitch variation profiles. The statistical parameters of this ensemble of pitch profiles were estimated by the structural TEM microscopy observations. This approach proved very successful in encompassing the many nonidealities arising at sample formation time, both in the longitudinal as well as in the transverse directions. The broadband reflective behavior for moderate annealing times was found due to a linear pitch transition zone between the initial blue and red films. Longer annealing times lead to a homogeneous pitch profile due to extensive thermal diffusion between the two films and, thus, to narrow-band reflection. The dispersive losses at shorter wavelengths observed experimentally were attributed to scattering losses between the domain walls and surfaces randomly formed inside the bulk of the untreated cholesteric films, as evidenced by the absence of any absorption, whatsoever, when the liquid crystal is in the isotropic state. A general theoretical study was attempted in order to provide some insight on the optimum pitch transition profile between two cholesteric films in terms of reflective efficiency; the linear transition proved most satisfactory among a set of representative transition profiles examined. Finally, a discrete slab film layout with enhanced reflective behavior was proposed as an alternative structure for broadband reflectors.

\section{ACKNOWLEDGMENTS}

The authors are grateful to Dr. F.-H. Kreuzer and Dr. E. Hanelt (Wacker Chemie $\mathrm{GmbH}$ ) for their kind support and the cholesteric compounds. They also thank Dr. N. Dessaud for her contribution in the early stages of this collaboration. D.C.Z. and E.E.K. acknowledge the financial support of the Greek General Secretariat of Research and Technology.
[1] P. de Gennes and J. Prost, The Physics of Liquid Crystals (Clarendon Press, Oxford, 1993).

[2] N. Boiko and V. Shibaev, Int. J. Polym. Mater. 45, 533 (2000).

[3] D. Broer, J. Lub, and G. Mol, Nature (London) 378, 467 (1995).

[4] D. Broer, G. Mol, A. van Haaren, and J. Lub, Adv. Mater. (Weinheim, Ger.) 11, 573 (1999).

[5] D. Coates, M. Goulding, S. Greenfield, J. Hanmer, S. Marden, and O. Parri, Proc. S.I.D., 1996, p. 67.

[6] D. Coates, M. Goulding, S. Greenfield, J. Hanmer, S. Marden, O. Parri, M. Verrall, and J. Ward (Proc. IDW 1996), p. 309.

[7] H. Guillard, P. Sixou, and V. Gandolfo, Project SMARTWIN, joule III programme, CT97-0068, mid-term report (1999).

[8] W. Schlichting, S. Faris, L. Li, B. Fan, and J. Kralik, Proc. SPIE 2690, 232 (1996).

[9] W. Schlichting, S. Faris, L. Li, B. Fan, J. Kralik, J. Haag, and Z. Lu, Mol. Cryst. Liq. Cryst. Sci. Technol., Sect. A 301, 231 (1997).

[10] Z. Lu, L. Li, H. Vithana, Y. Jiang, and S. Faris, Mol. Cryst. Liq. Cryst. Sci. Technol., Sect. A 301, 237 (1997).

[11] L. Li and S. Faris, Proc. S.I.D., 1996, p. 111.

[12] J. Kralik, B. Fan, H. Vithana, L. Li, and S. Faris, Mol. Cryst. Liq. Cryst. Sci. Technol., Sect. A 301, 249 (1997).

[13] W. Schlichting, S. Faris, B. Fan, J. Haag, Z. Lu, S. Kane, L. Li, T. Milster, and H. Luo, Jpn. J. Appl. Phys., Part 1 36, 587 (1997).

[14] M. Mitov, A. Boudet, and P. Sopéna, Project SMARTWIN, joule III programme, CT97-0068, first periodic progress report (1998).

[15] M. Mitov, A. Boudet, and P. Sopéna, Eur. Phys. J. B 8, 327 (1999).

[16] C. Binet, M. Mitov, and A. Boudet, Mol. Cryst. Liq. Cryst.
Sci. Technol., Sect. A 339, 111 (2000).

[17] A. Boudet, C. Binet, M. Mitov, C. Bourgerette, and E. Boucher, Eur. Phys. J. E 2, 247 (2000).

[18] S. Chen, R. Jin, D. Katsis, J. Mastrangelo, S. Papernov, and A. Schmid, Liq. Cryst. 27, 201 (2000).

[19] C. Binet, M. Mitov, and M. Mauzac, J. Appl. Phys. 90, 1730 (2001).

[20] A. Lavernhe, M. Mitov, C. Binet, and C. Bourgerette, Liq. Cryst. 28, 803 (2001).

[21] N. Leroux and L.-C. Chien, Proc. of the ACS Meeting, New Orleans, 1996, Vol. 37, p. 195.

[22] N. Leroux, W. Fritz, J. Doane, and L.-C. Chien, Mol. Cryst. Liq. Cryst. Sci. Technol., Sect. A 261, 465 (1995).

[23] D. Dyer, U. Schröder, K.-P. Chan, and R. Twieg, Chem. Mater. 9, 1665 (1997).

[24] R. Hikmet and H. Kemperman, Nature (London) 392, 476 (1998).

[25] R. Hikmet and H. Kemperman, Liq. Cryst. 26, 1645 (1999).

[26] E. Nouvet and M. Mitov, Mol. Cryst. Liq. Cryst. 413, 515 (2004)

[27] M. Mitov, E. Nouvet, and N. Dessaud, Eur. Phys. J. E 15, 413 (2004).

[28] F. Kreuzer, D. Andrejewski, W. Haas, N. Häberle, G. Riepl, and P. Spes, Mol. Cryst. Liq. Cryst. Sci. Technol., Sect. A 199, 345 (1991).

[29] C. Titus, P. Bos, J. Kelly, and E. Gartland, Jpn. J. Appl. Phys., Part 1 38, 1488 (1999).

[30] E. Kriezis and S. Elston, Opt. Commun. 177, 69 (2000).

[31] C. Brown, E. Kriezis, and S. Elston, J. Appl. Phys. 91, 3495 (2002).

[32] P. Yeh and C. Gu, Optics of Liquid Crystal Displays (Wiley, New York, 1999). 
[33] S. Stallinga, J. Appl. Phys. 85, 3023 (1999).

[34] Q. Hong, T. Wu, and S.-T. Wu, Liq. Cryst. 30, 367 (2003).

[35] Y. C. Yang, C.-S. Kee, J.-E. Kim, H. Y. Park, J. C. Lee, and Y. J. Chun, Phys. Rev. E 60, 6852 (1999).

[36] I. Hodgkinson, K. Wu, A. Lakhtakia, and M. McCall, Opt.
Commun. 177, 79 (2000).

[37] R. Azzam, Appl. Phys. Lett. 61, 3118 (1992).

[38] K. Robbie and M. Brett, J. Vac. Sci. Technol. A 15, 1460 (1997). 